\title{
Cloud ERP VS On-Premise ERP
}

\author{
Wiam Yahea Al Hayek \\ Instructor, Princess Alia University College \\ Al-Balqa Applied University \\ Jordan \\ Rasha Ahmad Abu Odeh \\ Assistant Instructor, Princess Alia University College \\ Al-Balqa Applied University \\ Jordan
}

\begin{abstract}
Currently, to make a massive difference in the company service and enables the company to compete in the market. Companies should have a system that manages its operations and accelerate customer service, and keeps the company ready for future enhancement to maintain business continuity. Before seven years and more, companies relied on a local custom ERP system, which is usually far from business best practices. Also, it follows business needs and does not force companies to develop and implement business best practices. This type of ERP system cannot maintain business continuity, and it will put the companies out of the market by the end of the day. That was why companies seek a readymade international ERP system that has been built to match business best practices. A few years back, all big ERP companies start issuing a cloud version of their ERP system to replace the on-premise version. So another ERP system selection challenge has been added, which is whether to go with the cloud version or on-premise version. In this paper, the author aims to help the companies decide on ERP implementation type; the first part of the article includes a theoretical part of cloud-based implantation, explaining the main issues and most common definitions of the cloud. The second part presents the difference between the cloud-systems and on-premises systems by defining each type of implementation and shows the needed vital factors when choosing between Cloud ERP or On-premise ERP. In addition to illustrating the pros and cons of On-Premise and Cloud ERP implementation types, the research results were finally displayed.
\end{abstract}

Keywords: Clouds, On-premise, ERP, Hybrid, Implementation, Cloud Storage.

\section{What is an Enterprise Resource Planning (ERP)}

Enterprise Resource Planning Systemis a management tool that integrates business processes into a unified and manageable workflow for social, financial, and computing resource monitoring.

https://theappsolutions.com/blog/cloud/cloud-vs-onpremise-erp.

ERP is a software system that integrates the organization's functional units all collaboratively, asit extends outside the organization as a resource and customer to involve them in the integration process, as in Figure 1.Furthermore, it requires a lot of money and time to publish because it is a large information system. Many systems failed because they didn't rely on well-studied plans and where they possess limited resources. 


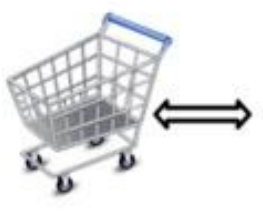

Customers

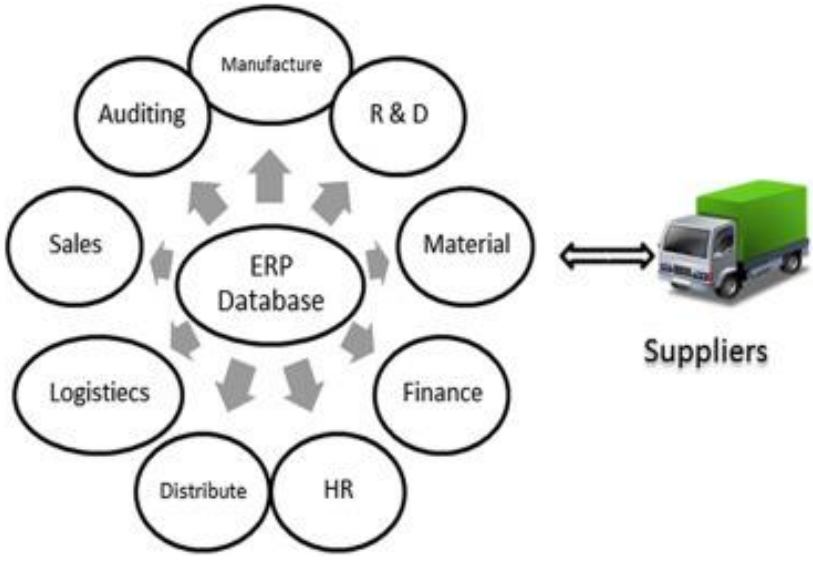

Figure 1

First of all, let us introduce the definition of cloud-based software and on-premise software in general.

Cloud-Based Software and On-Premise software

Cloud-based software means that you don't have the system at your premises, but you access your network using technological information resources that have been provided by a vendor based on predefined agreement. These technical information resources include the ERP system itself, servers installed, storage facilities, firewalls, security applications, and others. Sure, you should have internet access` to access all these resources, and no client installation is needed; usually, internet explorer is enough.

https://www.softwareadvice.com/resources/cloud-erp-vs-on-premise.

While on the other hand, On-premise software is installed locally on a company's computers and servers. The implemented software features may be accessed usually through client components installed on the client's machine. Web browsers can access some of the On-Premise functions and features.

Another implementation is called "Hybrid" deployments. This latter type is a mixture of the previously mentioned implementations in which cloud software is hosted on an organization's private servers.

A hybrid cloud solution is a solution that features an element of different types of IT deployment models, ranging from on-premises to private and public clouds. A hybrid cloud infrastructure depends on the availability of a public cloud platform supported by a trusted third-party provider, a private cloud constructedon-premises or through a hosted private cloud provider, and effective WAN connectivity between both of those environments.

https://www.cleo.com/blog/knowledge-base-on-premise-vs-cloud.

\section{Literature Review}

Demi and Haddara (2018) explained the updates on ERP within the organization to cloud-based systems and rethinking the traditional life cycle and showed that the infrastructure is different in the two systems and how ERP cloud systems affected ERP. The case of a cloud-based vendor has been studied in Norway, and their results indicated that ERP cloud systems have more extended time frames than those within the organization.

In another study by Megahed et al. (2019) entitled "Optimizing cloud solution design", the authors presented the importance of the cloud as well as building a cloud solution and encouraging companies to move from local environments to the public, private, or mixed cloud solutions where the requirements are provided to the user by the cloud service provider at low costs in a short time. In this case, problems are modeled by analyzing data, summarizing the problem, and programming it correctly to solve problems. By designing cloud solutions that meet customer requirements, cloud supply constraints, and solution access at lower costs and short time. the authors in this paper evaluated both approaches and demonstrated the effectiveness and efficiency of cloud solutions of all kinds (public, private, or hybrid) on local environments.

First, exceptional services can be accomplished to improve the quality of people's life by providing widely distributed systems in the cloud and mobile cloud deployments and having a decentralized structure based on an independent contract and resources without any specific centralized control and control. These cloud deployments can handle the provision of resources at a little cost, which will help expand many mobile applications and services that are willing to enlarge the range of billions of mobile devices without pain. 
It reflects examples of these data-based applications that bring popularity and profits and protect them initially, through the existing cloud structure and layers and provide a solution that deals with ample data storage. Second, it explores the use of the P2P cloud system (P2PCS) to process big data and analytics. Third, the cloud-based hybrid cloud model is proposed, and the model is applied to healthcare systems as a case study. Then, the model is simulated using a mobile cloud computing simulator (MCCSIM). Based on the power and trial delay results, the performance is up to $75 \%$ better than conventional cloud models. And finally, it can provide and analyze security and privacy countermeasures against potential attacks (Tawalbeh\&Saldamli, 2019).

The web services can be used in applications and through the Internet renews interest in providing ERP services. The web services are identified for enterprise customers whereby a unique system is allocated to meet their specific needs. ERP service is rented from sellers flexibly. The system can also provide a solution for automating the allocation of ERP services by creating web services. The proposed service installation method builds on the concept of a genetic algorithm with knowledge of web services extracted from the web service with raw group theory. A prototype of the system was built on the Google App Engine platform to verify the proposed installation. Based on the prototype operation results, it has proven its effectiveness and high ability to support the fully functional Cloud ERP platform (Chen et al., 2015).

The foremost vital factors need to be considered while choosing between Cloud ERP or On-premise ERP.

Does your business require a customized resource planning solution?

In Cloud ERP, customization is not an easy task and could be one of the significant reasons for the delay in the project implementation, especially when the vendor does not follow the best practice while customizing the application. While On-premise provides a more exceptional ability to be customized.

https://theappsolutions.com/blog/cloud/cloud-vs-onpremise-erp

ERP companies like Microsoft or Oracle put more restrictions on the cloud ERP customization to maintain their ERP system's unified identity. Also, they force partners and customers to have a test environment where any customization should be published and tested on it; then it can be published on the production environment after making sure that there is no conflict with the original ERP code and functions. Core ERP business logic doesn't allow partners to customize or extend it to ensure the system will keep working as expected. They provide what we called models and data models, where any customization can be maintained in these models and can be removed at any time without affecting original ERP system functions and features.

These restrictions take a long time, which increases the ERP project implementation timeline and, in feature, if the customer face any issue on the system and this issue required code changes (technical not functional issue), the response time may need days to fix the problem. While in an on-premise ERP system, fixing such technical issues will usually be faster within hours.

Full corporate business-critical data control is required?

In cloud ERP your data will be hosted outside your premises and managed by your vendor who can access whether you agreed or not!

Honestly, no one can guarantee that your customer list or critical financial data will not be used by the vendor or other parties that have an authority on the vendor; this is one of the main reasons for Government or military institutes not to utilize cloud facilities.

Losing data is not an issue these days because vendors apply various encryption and access management protocols and managed the backup and system high availability.

On-premise, the company has full control over data. Consequently, the company needs to be cautious about its data security policies and efficiency to avoid possible breaches and malicious attacks.

The scope of operation requires significant scalability capacity?

In Cloud ERP, benefits from cloud auto-scaling features allow taking as many resources as required to maintain operations. In the cloud, you can increase the number of servers, storage capacity, memory, resources utilization hours, or some users that can access cloud resources within minutes.

On-premises, scalability capacity is limited to the hardware. So if there is a plan for business growth, it should be aligned with the system expanding plan.

Criterial (Advantages and disadvantages) of On-Premise and Cloud Types of ERP

List of the main criteria of the two types of ERP deployment that companies should consider before taking the implementation type decision. 


\section{Cost-Effectiveness}

Cloud software is priced under a monthly or annual subscription, with additional recurring fees for support, training, and updates. On-premise software is generally priced under a one-time perpetual license fee, usually based on the organization's size or the number of concurrent users. There are recurring fees for support, training, and updates.

Cloud-based systems are considered an operating expenditure and other expenses; an additional overhead cost the organization will continue to pay. On-premise, the system cost is generally considered a capital expenditure, and the cost will depreciate in the coming years.

Cloud-based systems, companies need to pay for the resources depends on the consumption, but they will not pay for maintenance and upkeep costs. Companies that decide to deploy on-premise ERP systems are responsible for the servers hardware's ongoing costs, need operating system licenses, firewalls, antiviruses, power consumption, and space.

The company should consider the current used vertical solutions and standalone applications, which can be replaced by one ERP module or functions. If this is possible, cloud ERP will save all expenses related to vertical and standalone solutions, especially because most cloud ERP systems added a lot of modules and features compared with the onpremise versions. Examples include document archiving, email integration, workflows and alerts, the embedded mobile application that can be used to review and create system transactions, and no need for any additional license or remote software to access the system remotely.

For cloud-based implementation, the return of investment (ROI) will come after five years and depend on the investment size. In other words, the company will spend more or at least the same as an on-premise implementation during the first few years. However, the company will start gaining profits after that, especially if there is a need for the scaling system functionality or the required hardware.

Cloud: In general, it is considered a low-cost system, and costs are expected over time. The downside is that the organization may spend more money over the life cycle of the system. In Prem: It has a high initial cost and is more dangerous. Institutions are required to pay the cost of ongoing maintenance, hardware, and IT personnel costs (Hughes, 2018).

\section{Implementation}

Cloud implementation generally shorter than on-premise implementation. There is no installation required, no hardware purchasing. Customer business needs almost can be covered by out of the box system functions, so no customization is needed; this will make implementation faster.

Usually, the vendor informs the customer that all provided solutions for his business needs will be covered by a system out of the box functions or using a workaround but not customizing the system. Therefore, it canbe challenging to find a cloud-based solution to fit all the needs of an implementing organization (Duan et al, 2013), as mentioned previously, because of the limitations and the difficulties of cloud-based ERP customization.

In On-premise, the company entirely handles the implementation of on-premise ERP. Because of this, the process takes a considerable amount of time. Companies should consider needed time for infrastructure preparation, servers, and required software licenses, the system installation, and hiring of needed IT staff, or purchasing IT services from vendors to handle all required IT tasks.

Both Cloud and On-premise ERP systems provide tools to facilitate and accelerate master data upload. But in the cloud version, these tools much better than on-premise, and it covers more entities. Cloud versions of some ERP systems have a Master Data Management tool that makes sure that all data are consistent, and there is no missing data. So the user will not face any missing setup data issue when start using the system.

The time of implementation, generally for both options, depends on the modules that need to be implemented and the company and implementer (vendor).

\section{Customization}

Cloud-based ERP service is usually bound to its features set but enables customization to a certain extent for an additional fee. The way of uploading customization to the cloud-based ERP has many verification steps. Uploading will ensure that customization matches the system best practice, and there will be no conflict with the original system functions. Some cloud-based ERP considers the test environment and development environment crucial, so people cannot customize without building it on the development environment. 
The building will thenbe followed by testing it using the test environment. After that, this customization can be applied to the production environment. These restrictions are time-consuming impact, which will be reflected in the cost.

On-premise ERP is open for all sorts of customization, according to the business needs. After all, the assets are all there, and people can rearrange them to fit their needs. However, this comes with additional spending and may cause various operational setbacks like prolonged downtime or accidental misconfiguration.

\section{https://www.softwareadvice.com/resources/cloud-erp-vs-on-premise}

Customization in on-premise ERP is secure, and in most ERP systems can be applied to the production environment immediately.

\section{Scalability}

Cloud ERP benefits from cloud auto-scaling features that allow taking as many resources as required to maintain operation. In addition to hardware scalability, there is a lot of tested application that can be activated on the cloud ERP system and implemented within days or weeks. On-premise ERP's scalability capacity is limited to the hardware. These constraints mean one needs to plan growth and expand the system accordingly (Buyya et al., 2013).

\section{Maintenance}

Cloud ERP's vendor handles the operation on its own and regularly updates its features and security framework. The company requires minimal maintenance staff to oversee implementation and integration - otherwise, it is all about using the application without worrying about its inner workings.

On-premise ERP, the company is responsible for the whole thing. This aspect results in the need to train or hire specialized staff for deployment and tech support.

https://www.softwareadvice.com/resources/cloud-erp-vs-on-premise

Cloud ERP's high availability is maintained by the vendor and depends on cloud resource accessibility time if its 24 hours over seven days or not, and the company does not need to worry about data backup because the vendor handles it.

On-premise ERP, companies should provide all tools, hardware, and software licenses required to maintain system high availability and secure the data. Data backup part of the central and critical tasks for the company.

\section{Conclusion}

When it comes to select a new ERP system, there are more ERP options than ever for businesses of all sizes. Companies should carefully consider all mentioned points because ignoring any may cause the wrong decision will cost too much.

Companies should study each mentioned point separately. So I assumed that the company should know:

ERP project budget, this is a crucial point in some cases, plays the main rule in making ERP implementation decisions regardless of other point evaluations.

- How much precisely the cost of the current system, the cost should include at least listed main points:

$\checkmark$ License and enhancement fees

$\checkmark$ support contract cost

$\checkmark$ power consumption

$\checkmark$ IT staff cost

$\checkmark$ Active standalone applications cost in addition to the integration cost

$\checkmark$ Additional user cost

$\checkmark$ System changes cost

- What are the current modules that need to be implemented

- What are the future modules that need to be implemented

- What are the standalone applications and the vertical solutions that can be replaced by the standard ERP module?So the renewed standalone and vertical applications should be eliminated when the company make the cost comparison

- Under any situation, what is the impact of sharing company critical data if it exists 
- Based on the company vision and mission. When there will be a business expand and if this requires new modules to be implemented or increasing the number of employees who will use the system and required additional licenses

- Do I have a lot of special business needs that will never match other business operations best practices? So to meet these unique needs, I customize the system

- Type and cost of these customizations. Is it minor, major, or core changes?

- There is a need to access the system remotely.

- High system availability is a must.

- There is any need to integrate the system with other cloud services.

In general, benefits from cloud computing Fast completion, soft update, lower cost, scalability, improved accessibility, and high availability. From studying various researches, there are other benefits such as the use of advanced technology, rapid updates, easy application, improved accessibility, improved mobility, and easier integration with services Cloud, cost transparency, sales automation, plus security and free trial(Abd Elmonemet al., 2016).

Conversely, on-premise ERP systems offer advantages in customization and control but are more expensive upfront, and many do not support mobile. This case can be problematic for smaller buyers, but, as is usually the case, it depends on the specific needs of the individual business.

\section{References}

Abd Elmonem, M., Nasr, E. \&Geith, M. (2016). Benefits and challenges of cloud ERP systems - A systematic literature review, Future Computing and Informatics Journal, Vol. 1, Issues 1-2, pp.1-9.

Buyya, R. Vecchiola, C., \&Selvi, S. (2013). Mastering cloud computing (1st ed.), Elsevier

Chen, C., Liang, W., Hsu, H. (2015).“A cloud computing platform for ERP applications”, Applied Soft Computing, Vol. 27, pp.127-136.

Demi, S., Haddara,M. (2018).“Do Cloud ERP Systems Retire? An ERP Lifecycle Perspective,", Procedia Computer Science,Vol. 138, pp. 587-594.

Duan, J., Faker, P., Fesak, A., \& Stuart, T. (2013). Benefits and drawbacks of cloud-based versus traditional ERP systems. Proceedings of the 2012-13 course on Advanced Resource Planning.

Hughes, A. (2018). Cloud Computing vs. On-Premises: The Differences of Both Platforms, retrieved from https://www.cleo.com/blog/knowledge-base-on-premise-vs-cloud.

Klaus, H., Rosemann, M., \& Gable, G. (2013). What is ERP? Inf Syst Front, 2 (2000), pp. 141-162

Lenart, A. (2011). editor ERP in the Cloud-Benefits, and Challenges. EuroSymposium on Systems Analysis and Design; Springer.

Megahed, A., Nazeem, A., Yin, P., Tata, S., Reza, H., Nezhad, M., \&Nakamura, T. (2019).“Optimizing cloud solution design," Future Generation Computer Systems, Vol. 91, pp. 86-95.

Peng, G., Gala C. (2014). Cloud ERP: a new dilemma to modern organizations? Journal of Computer Information Systems, 54(4), 22-30

Tawalbeh, L.\&Saldamli G. (2019). "Reconsidering significant data security and privacy in cloud and mobile cloud systems," Journal of King Saud University - Computer and Information Sciences, "Available online 29 May 2019

https://en.wikipedia.org/wiki/On-premises_software

https://theappsolutions.com/blog/cloud/cloud-vs-onpremise-erp.

https://www.softwareadvice.com/resources/cloud-erp-vs-on-premise.

https://www.cleo.com/blog/knowledge-base-on-premise-vs-cloud. 